\title{
Focus on Myelodysplastic Syndromes Reviewed
}

\author{
Guido D'ANGELO \\ Laboratory of Clinical-Chemistry, Hematology and Microbiology, ASST - Gallarate Hospital, Varese - ITALY
}

\begin{abstract}
Myelodysplastic syndromes (MDSs) are neoplastic pathologies characterized by accelerated hematopoiesis and apoptosis; this results in a paradoxical condition featured by bone marrow progenitors hypercellularity compared to the peripheral cytopenia. In this review the WHO update goal is summarized. A new MDS nomenclature has been defined so as to make the diagnostic approach towards these heterogeneous diseases more rational. Methodologically, the morphology study is still considered fundamental, both for the accurate blasts counting and the dysplastic detection of one or more of the three myeloid lines. Furthermore, specific genetic mutations have not only a diagnostic and prognostic importance, but it can also define a more adherent therapeutic condition, as well as identifying blurred categories or cytopenic asymptomatic patients who can evolve towards an overt MDS.
\end{abstract}

Keywords: Myelodysplastic syndrome, WHO, Classification, Blasts, Genetic mutation

\section{INTRODUCTION}

Myelodysplastic Syndome(s) (MDSs) are clonal disorders characterized by intramedullary simultaneous proliferation and apoptosis of hematopoietic cells. ${ }^{1}$ The result is an ineffective hematopoiesis that results cytopenia(s) (hemoglobin $(\mathrm{Hb})<100 \mathrm{~g} / \mathrm{L}$, absolute neutrophil count $(\mathrm{ANC})$ $<1.8 \times 10^{9} / \mathrm{L}$, platelets (PLTs) $<100 \times 10^{9} / \mathrm{L}$, according to the International Prognostic Score System (IPSS). ${ }^{2}$ The values should be considered not exclusionary). Another important diagnostic requirement for MDS is the dysplasia that can be present in one or more of the three myeloid lines. It should be emphasized that the myeloid term includes all cells belonging to the granulocytic, monocytic/ macrophage, erythroid, megakaryocytic and mastcells lineages. Finally, the inevitable risk that the MDS evolves towards an acute myeloid leukemia (AML).
The purpose of this review is to provide an easy-touse tool for diagnosing MDS. These heterogeneous pathologies, which mainly affect the elderly, can show an overlap with myeloproliferative neoplasms (MDS/MPN) in same patients, while other patients may not show overt characteristics of a pre-leukemic condition.

\section{Rational diagnostic approach for MDS Classi- fication}

According to the WHO classification ${ }^{3}$, cytochemistry, immunophenotype, genetics and clinical features are the key methods to define the MDS as a clinically significant disease entities (Table 1). The employment of the aforementioned methods allows the classification of MDS that can be used in daily clinical practice, and can serve as common language for clinical trials and laboratory investigation. 
International Journal of Hematology and Oncology

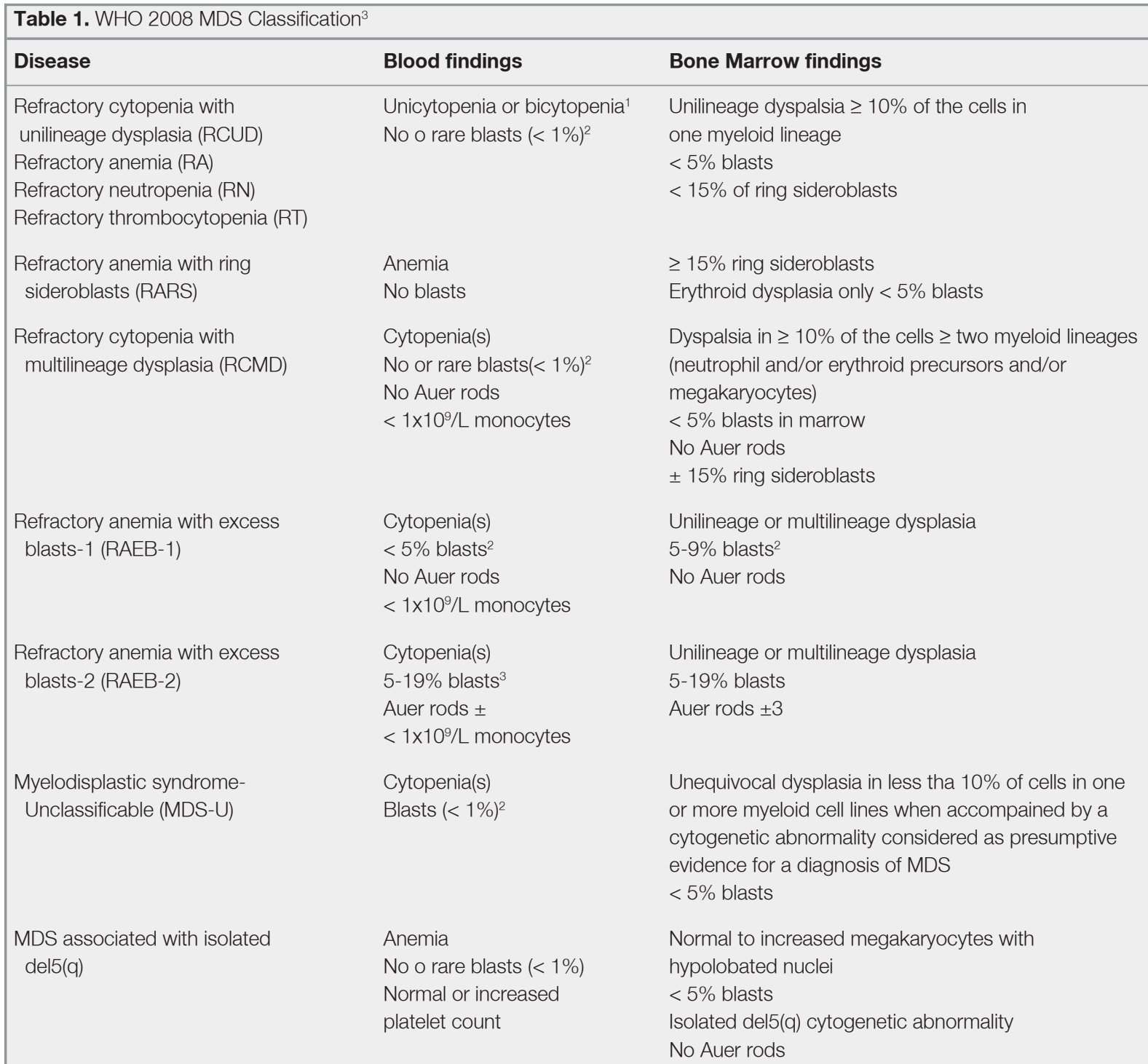

${ }^{1}$ Bicytopenia may occasionally be observed. Cases with pancytopenia shuld be classified as MDS-U

2 If marrow myeloblasts percentage is $<5 \%$ but there are $2-4 \%$ myeloblasts in the blood, the diagnostic classification is RAEB- 1 . Cases with RCUD and RCMD with $1 \%$ myeloblasts in the blood should be classified as MDS-U.

${ }^{3}$ Cases with Auer rods $<5 \%$ blasts in the blood and $<10 \%$ in the marrow should br classified as RAEB- 2 .

\section{WHO MDS Revised Nomenclature}

In 2016, the WHO proposed a MDS updates on nomenclature, morphology, immunophenotype, cytogenetic and gene mutations. ${ }^{4}$

Regarding the nomenclature, the WHO 2016 MDS revised, proposed a classification based on the morphological aspects of dysplasia, as well as the accurate count of blasts, not mainly on cytopenia (Table 2).

For categorizing and evaluating the disease progression, the blasts percentage $\geq 20 \%$, in both peripheral blood $(\mathrm{PB})$ and bone marrow $(\mathrm{BM})$, remains fundamental.

Regarding Refractory cytopenia with unilineage dysplasia (RCUD), it has been established that the type of dysplasia does not always fit the cytopenic cell line. Refractory Anemia (RA), Refractory Neutropenia (RN), and Refractory Thrombocytopenia (RT) subgroups are removed and the RCUD is incorporated into a single renamed group: MDS with single lineage dysplasia (MDS-SLD). 


\begin{tabular}{|c|c|}
\hline WHO MDS classification ${ }^{3}$ & 2016 WHO revised nomenclature ${ }^{4}$ \\
\hline $\begin{array}{l}\text { Refractory cytopenia } \\
\text { with unilineage dysplasia (RCUD) } \\
\text { - Refractory anemia (RA) } \\
\text { - Refractory neutropenia (RN) } \\
\text { - Refractory thrombocytopenia (RT) }\end{array}$ & $\begin{array}{l}\text { MDS with single lineage dysplasia } \\
\text { (MDS-SLD) }\end{array}$ \\
\hline RA with ring sideroblasts (RARS) & $\begin{array}{l}\text { MDS with ring sideroblasts } \\
\text { with single lineage dysplasia } \\
\text { (MDS-RSSLD) }\end{array}$ \\
\hline $\begin{array}{l}\text { Refractory cytopenia with } \\
\text { multilineage dysplasia (RCMD) }\end{array}$ & $\begin{array}{l}\text { MDS with ring sideroblasts } \\
\text { with multilineage dysplasia } \\
\text { (MDS-RSMLD) }\end{array}$ \\
\hline $\begin{array}{l}\text { Refractory anemia } \\
\text { with excess of blasts (RAEB) } \\
\text { - Refractory anemia with } \\
\text { excess of blasts-1 (RAEB-1) } \\
\text { - Refractory anemia with } \\
\text { excess of blasts-2 (RAEB-2) }\end{array}$ & $\begin{array}{l}\text { MDS with excess of blasts } \\
\text { (MDS-EB) } \\
\text { - MDS-EB1 } \\
\text { - MDS-EB2 }\end{array}$ \\
\hline MDS with isolated del(5q) & MDS with isolated del(5q) \\
\hline MDS unclassifiable & MDS unclassifiable \\
\hline $\begin{array}{l}\text { Childhood MDS } \\
\text { - Refractory cytopenia of childhood }\end{array}$ & $\begin{array}{l}\text { Childhood MDS } \\
\text { - Refractory cytopenia of childhood }\end{array}$ \\
\hline
\end{tabular}

Refractory anemia with ring sideroblasts (RARS) is reclassified as MDS with ring sideroblasts with single lineage dysplasia (MDS-RSSLD). Refractory cytopenia with multilineage dysplasia (RCMD), with the new nomenclature, is divided into two groups, MDS with ring sideroblasts with multilineage dysplasia (MDS-RSMLD) and MDS with multilineage dysplasia (MDS-MLD).

The nomenclature for refractory anemia with excess of blasts (RAEB) changes in MDS with excess of blasts (MDS-EB), as well as the RAEB-1 and RAEB-2 subgroups that are renamed MDSexcess of blasts-1 (MDS-EB-1) and MDS-excess of blasts-2 (MDS-EB-2) respectively.
The nomenclature of MDS with isolated del(5q), unclassifiable MDS and childhood MDS with the subgroup refractory cytopenia of childhood does not change.

\section{Distinctive Morphological Feature of MDS Dysplasia}

Within the new WHO classification, $10 \%$ remains the cut-off detention of dysplastic line.

Cellular characteristics of dysplastic morphology

The features of dysplastic cellular morphology ${ }^{5}$ are referred to nuclear and cytoplasmic dyserythropoiesis, disgranulopoiesis in myeloid-granulocytic line, and dismegakaryocytopoiesis ${ }^{6}$ (Table 3 ).

\footnotetext{
Table 3. Cellular characteristics of dysplastic morphology

Dyserythropoiesis nuclear Nuclear budding, internuclear bridging, karyorhexis, multinuclearity, nuclear hyperlobation, megaloblastic changes

Dyserythropoiesis cytoplasmatic Ring sideroblasts, vacuolization, periodic acid-Shiff positivity.

Disgranulopoiesis

Small or unusually large size, nuclear hypolobation (pseudo Pelger-Huët, pelgeroid), irregular hypersegmentation, decreased granules, agranularity, pseudo Chediak-Higashi granules, Auer rods.

Dismegakaryocytopoiesis

Micromegakaryocytes, nuclear hypolobation, multinucleation (normal megakaryocytes are uninucleate with lobulated nuclei
} 


\begin{tabular}{|ll|}
\hline Table 4. Immunophenotype assay. & Main monoclonal antibodies employed for studying hematopoietic dysplastic lines \\
\hline Hematopoietic line & Monoclonal antibodies \\
\hline Myeloid & CD16, CD11b: neutrophilic granulocytes undergoing maturation \\
& CD13/CD16 combination: neutrophilic maturation pattern \\
& CD2, CD5, CD7, and CD19, monoclonal antibodies belonging to lymphoid lineage, \\
& can be expressed abnormally both myeloid progenitors and maturing myeloid cells \\
& CD34 \\
Blast Cells & Most CD34+ blast cells are committed towards the myeloid lineage (CD38+, \\
& HLA-DR+, CD13+, CD33+) \\
Monocytic & CD56, HLA-DR, CD36, CD33, CD15, CD14, CD13, and CD11b \\
Erythroid & Glycophorin A (Gly A), CD71 \\
& Between the two monoclonal antibodies, the Gly A does not allow the detention of \\
& erythroid precursors more immature \\
Megakaryocytic and Platelets & CD61, CD41 \\
\hline
\end{tabular}

In absence of genetic abnormalities, the diagnosis of MDS is primarily based on the presence of dysplasia in PB and BM cells.

\section{Blasts}

The threshold of blasts, the $2 \%$ cut-off, which was introduced by the International Prognostic Score System-Rivisited (IPSS-R) ${ }^{7}$, is difficult to interpret because the distinction between blasts categories $0-2 \%$ vs $>2 \%$ vs $<5 \%$ is not easily reproducible. For this reason, to report the exact number of blasts morphologically detected is recommended, rather than "blasts $<5 \%$ ".

\section{Blasts $<20 \%$ and AML Diagnosis}

With the percentage of blasts $<20 \%$, cytogenetic investigation for abnormal karyotype detection and/or genetic investigation for somatic mutations are crucial for the presence of $\mathrm{t}(8 ; 21)(\mathrm{q} 22 ; \mathrm{q} 22)$, $\operatorname{inv}(16)(\mathrm{p} 13.1 ; \mathrm{q} 22)$ or $(16 ; 16)(\mathrm{p} 13.1 ; \mathrm{q} 22)$, and RUNX1-RUNX1T1 mutation.

CBFB-MYH11 or PML-RARA detection is already a diagnostic condition for AML, regardless of the blasts percentage.

The detection of other genetic abnormalities, such as $\mathrm{t}(9 ; 11)(\mathrm{p} 21.3 ; \mathrm{q} 23.3) \quad$ KMT2AMLLT3, $\mathrm{t}(6 ; 9)$ (p23;q34.1) DEK-NUP214 and NPM1 mutations are still controversial.

\section{MDS-Unclassified (MDS-U), 2016 WHO Diag- nostic Criteria}

MDS-U with single or multiline dysplasia and blasts $<5 \%$ into $\mathrm{BM}$, but $1 \%$ blasts into $\mathrm{PB}$, the WHO recommends the blasts detection in $\mathrm{PB}$ at least in two separate investigations.

In MDS with unilinear dysplasia but pancytopenia, the $\mathrm{Hb}, \mathrm{ANC}$ and PLT values must be lower than the reference values indicated by IPSS.

\section{Acute erythroid leukemia (AEL) (type erythroid/} myeloid), 2016 WHO classification

The myeloid malignancy with erythroid precursors $\geq 50 \%$ within nucleated cells in BM and myeloblasts percentage in $\mathrm{BM}$ (or $\mathrm{PB}$ ) $<20 \%$, but $\geq 20 \%$ compared to non-erythroid cells, was classified as AEL (erythroid/myeloid subtype)-AML-not otherwise specified (AML-NOS). The 2016 WHO classification identifies these cases as MDS-EB, as it is shown that acute erythroleukemia patients have similar risk-adjusted outcome to RAEB patients, and they do not seem to gain survival advantage with acute myeloid leukemia-type induction chemotherapy. ${ }^{8}$ Pure erythroid leukemia remains as a subtype of AML-NOS.

\section{Immunophenotyping in MDS}

Although the flow cytometry immunophenotype (FCI) analysis is an accurate method for qualitative and quantitative evaluation of hematopoietic cells, it alone does not allow the diagnosis of MDS. 


\begin{tabular}{|ll|}
\hline Table 5 : Main somatic mutations and more frequent alterations \\
\hline Mutation & Associated alterations \\
\hline N-RAS/K-RAS & Associated with thrombocytopenia, blast excess, monocytosis, is most common in CMML \\
RUNX1 & Associated with thrombocytopenia and excess blasts \\
ASXL1 & Is a frequent molecular aberrations in MDS and predict an adverse prognostic outcome \\
EZH2 & Very common in CMML \\
TP53 & Associated with complex and monosomal karyotype, excess blasts, thrombocytopenia, \\
ETV6 & few mutation in other genes \\
DNMT3A & Is a common additional abnormality in patients with myelodysplastic syndromes or acute \\
U2AF1 & myeloid leukemia and monosomy 7 \\
& Occur early in the course of MDS \\
\hline
\end{tabular}

Since in MDS the hematopoietic cells immunophenotype is characterized by the abnormal expression of different cell antigens, FCI analysis must be reproducible between different operators and the result comprehensible for the clinicians. ${ }^{9}$ In addition, the limited availability of markers for the erythroid line, does not always make the evaluation of erythroid line dysplasia easy. ${ }^{10}$

FCI can be considered "supportive" for diagnosing MDS and the results must always be integrated with the BM report morphology. When morphology and, mainly, cytogenetics are cogent, an abnormal FCI can be helpful to diagnose MDS.

In Table 4 the main monoclonal antibodies employed about the hematopoietic dysplastic lines study are summarized.

\section{Genetics in MDS}

Predicting prognosis is important for defining the risk and treatment options. Abnormalities of karyotype, considered in the current MDS prognostic score, are present in less than $50 \%$ of cases. In contrast, somatic mutations associated with the disease have been shown to be much more adherent to support their employment as a prognostic biomarker. Mutations of N-RAS/K-RAS, RUNX1, ASXL1, EZH2, TP53, ETV6, DNMT3A, U2AF1, identify patients with poor prognosis, compared to their non-mutated counterparts (Table 5). Like the karyotype, patients with multiple mutations show advanced disease, high mortality risk, and AML transformation. ${ }^{11}$

In MDS-RSSLD and MDS-RSLD, whose diagnoses are made on the presence of ring sideroblasts percentages $\geq 15 \%$ or $\geq 5 \%$ in presence of SF3B1 mutation respectively, the SF3B1 gene spliceosome mutation is present in more than $70 \%$ of cases. In the presence of blasts, the diagnosis of MDS-RSSLD is excluded. If multilineage dysplasia without a blast cell increase is present, a case is classified as MDS-RSMLD.

\section{MDS with isolated del( $5 q)$}

For the purposes of diagnosis, $\operatorname{del}(5 q)$ is the only cytogenetic abnormality that must be present; except for monosomy 7, the 2016 WHO classification does not allow other cytogenetic alterations.

In MDS with isolated del(5q), which generally has a favorable prognosis, the TP53 mutation evaluation and/or p53 detection by immunohistochemistry is recommended. ${ }^{12}$ In about $20 \%$ of patients with del $(5 q)$ and early disease stage, the mutation is present. It identifies a subgroup with adverse prognosis for increased risk of evolution in AML and resistance to lenalidomide therapy. ${ }^{13}$

\section{Blurred categories of MDS, Myeloproliferative Neoplasm and/or Acute Leukemia}

These are conditions in which clinical and/or diagnostic aspects can show a blurred diagnostic frame- 
work, such as the prevalence of cytopenia, in one or more cell lines, respect to dysplasia, or absence of karyotype abnormalities but presence of somatic specific mutations for hematological pathology but without signs of illness.

Idiopathic Cytopenias of Undetermined Significance (ICUS): cytopenia should be persistent in one or more lines for at least six months, with no other pathologies justifying it, and no other diagnostic criteria that meet MDS diagnosis according to WHO. ${ }^{14}$ Some patients show long-lasting cytopenia stability without complications, other patients may have an alternative diagnosis, or evolve towards an open MDS or AML. ${ }^{15}$ Patient observation is recommended.

Clonal Cytopenia of Undetermined Significance (CCUS): in cytopenic patients CCUS is a more frequent diagnosis than MDS. In these patients the clonality can explain cytopenia, but the number of mutations, as well as the type, do not seem to have a clear significance prognostic yet. Clinical recognition of CCUS will define it as a formally defined diagnostic entity. ${ }^{16}$

Clonal Hematopiesis of Indeterminated Potential (CHIP): generally present in apparently healthy elderly patients, it is characterized by the presence of clonal mutations identical to those found in MDS, and the evolution is towards an open MDS.

Thirty-five percente of ICUS carry MDS-associated somatic mutations and these patients can be identified as CCUS. CCUS and MDS patients who share similar mutations may have diagnostic relevance..$^{17}$ However, the presence of isolated MDS somatic mutations associated with unexplained cytopenia is not considered diagnostic for MDS.

\section{Reactive Cytopenia and Dysplasia}

It is known that some conditions, such as vitamin $\mathrm{B}_{12}$ and/or folate deficiency, HIV infection, copper deficiency ${ }^{18}$, alcohol abuse, and drugs (e.g. methotrexate) can cause cytopenia and dysplasia. In addition, it should be stressed that in some ethnic groups, the neutrophils absolute reference value is lower then Caucasian, as well as the platelets value. ${ }^{19}$ Then, in these cases the isolated neutropenia should be well interpreted.
In some normal subjects, dysplasia can be more than $10 \%$, but it is generally limited to a single cell line and without preponderant morphological aspects. Moreover, the presence of cytopenia is not due to neoplastic conditions.

In the elderly, TET2 mutations can be detected with acquired clonal hematopoiesis. In addition to TET2 mutation, DNMT3A, ASXL1 and SF3B1 mutations may represent a pre-malignant conditions that cause a clonal hematopoietic expansion.

\section{Discussion and Conclusion}

In oncohematology, morphology still plays an important role as a "first engine", mainly in these hematologic neoplasms in which the detection of typical morphological aspects, as well as the accurate counting of blasts are crucial for starting further investigation and which have a significant impact on overall patient management.

The fundamental contribution of genetic mutations, in addition to cytogenetics for a MDS diagnosis, allows us to define the prognosis and, consequently, to select patients for a more appropriate therapy. Moreover, family germ line mutations have allowed us to identify patients subgroups in which family study is justified for certain categories of genes (e.g. CEPBA, DDX41, RUNX1, ANKRD26, ETV6, GATA2)20.

\section{REFERENCES}

1. Scheinberg $P$, DeZern AE, Steensma DP. Acquired marrow failure syndromes: aplastic anemia, PNH and MDS. American Society of Hematology Self-Assessment Program Textbook 2016: 478-520.

2. Greenberg P, Cox C, LeBeau MM, et. al. International Scoring System for evaluating prognosis in myelodysplastic syndromes. Blood 89: 2079-2088, 1997.

3. Swerdlow SH, Campo E, Harris NL, et al, eds. WHO classification of tumours of haematopoietic and lymphoid tissues. Lyon, France: IARC; 2008.

4. Arber DA, Orazi A, Hasserjian R, et al. The 2016 revision to the World Health Organization classification of myeloid neoplasms and acute leukemia. Blood 127: 2391-2405, 2016.

5. Invernizzi R, Quaglia F, Della Porta MG. Importance of classical morphology in the diagnosis of myelodysplastic syndrome. Mediterr J Hematol Infect Dis 7: e2015035, 2015. 
6. Goasguen JE, Bennett J, Bain BJ, et al. Quality control initiative on the evaluation of the dysmegakaryopoiesis in myeloid neoplasms: Difficulties in the assessment of dysplasia. International Working Group on Morphology of MDS IWGM-MDS. Leuk Res 45: 75-81, 2016.

7. Greenberg PL, Tuechler H, Schanz J et al. Revised International Prognostic Scoring System (IPSS-R) for myelodysplastic syndromes. Blood 120: 2454-2465, 2012.

8. Wang SA, Patel KP, Pozdnyakova O, et al. Acute erythroid leukemia with $<20 \%$ bone marrow blasts is clinically and biologically similar to myelodysplastic syndrome with excess blasts. Modern Pathology 29: 1221-1231, 2016.

9. Della Porta M.G., Picone C. Diagnostic utility of flow cytometry in myelodysplastic syndromes.. Mediterr J Hematol Infect Dis 9: e2017017, 2017.

10. Della Porta MG, Malcovati L, Invernizzi R, et al. Flow cytometry evaluation of erythroid dysplasia in patients with myelodysplastic syndrome. Leukemia 20: 549-555, 2006.

11. Papaemmanuil E, Gerstung M, Malcovati L, et al. Clinical and biological implications of driver mutations in myelodysplastic syndromes. Blood 122: 3616-3627, 2013.

12. McGraw KL, Johnny Nguyen J, Komrokji RS, et al. Immunohistochemical pattern of p53 is a measure of TP53 mutation burden and adverse clinical outcome in myelodysplastic syndromes and secondary acute myeloid leukemia. Haematologica 101: e320-e323, 2016.

13. Pellagatti $\mathrm{A}$, Boultwood $\mathrm{J}$. The molecular pathogenesis of the myelodysplastic syndromes. Eur J Haematol 95: 3-15, 2015.

14. Malcovati L, Cazzola M. The shadowlands of MDS: idiopathic cytopenias of undetermined significance (ICUS) and clonal hematopoiesis of indeterminate potential (CHIP). Hematology 2015: 299-307, 2015.

15. Scheinberg P, DeZern AE, Steensma DP. Acquired marrow failure syndromes: aplastic anemia, PNH and MDS. American Society of Hematology Self-Assessment Program textbook. 2016: 478-520.
16. Kwok B, Hall JM, Witte JS, et al. MDS-associated somatic mutations and clonal hematopoiesis are common in idiopathic cytopenias of undetermined significance. Blood 126: 23552361, 2015

17. Steensma DP, Bejar R, Jaiswal S, et al. Clonal hematopoiesis of indeterminate potential and its distinction from myelodysplastic syndromes. Blood 126: 9-16, 2015.

18. D’Angelo G. Copper deficiency mimicking myelodysplastic syndrome. Blood Res 51: 217-219, 2016.

19. D'Angelo G. Ethnic and genetic causes of neutropenia: clinical and therapeutic implications. Laboratory Hematology 15: 25-29, 2009.

20. West AH, Godley LA, Chumpek JE. Familial myelodisplastic syndrome/acute leukemia syndromes: a review and utility for translational investigations. Ann N Y Acad Sci 1310: 111-118, 2014.

\section{Correspondence:}

Guido D'ANGELO, M.D.

Hematology/Coagulation

Laboratory of Clinical-Chemistry, Hematology and

Microbiology

ASST- Gallarate Hospital

Varese / ITALY

Tel. +390331. 751456

Fax +390331.751789

e-mail: danguido@libero.it 